\title{
100 Jahre Betriebsrätegesetz und aktuelle Partizipation von Beschäftigten in Deutschland
}

\author{
Am 4. Februar 1920 trat in Deutschland das erste Betriebsrätegesetz in Kraft. Nachdem es \\ 1934 ausgesetzt worden war, führte der Alliierte Kontrollrat die betriebliche Mitbestimmung \\ 1946 wieder ein. Nach mehreren Reformen hat sich die Mitbestimmung etabliert. Während \\ aber in Großunternehmen die betriebliche Mitbestimmung fest verankert ist, wird im \\ Mittelstand eher auf weniger institutionalisierte Beteiligungsformen gesetzt. Der Wandel \\ der Arbeitsorganisation durch Crowdworking, Telearbeit und Solo-Selbständigkeit stellt \\ allerdings die betriebliche Zusammenarbeit vor neue Herausforderungen. Derzeit ist noch nicht \\ erkennbar, dass die Betriebsräte darauf nicht mithilfe der bereits in der Betriebsverfassung \\ angelegten Befugnisse angemessen reagieren können. Das Jubiläum sollte Anlass dafür \\ sein, intensiver über die Zukunft der deutschen Betriebsverfassung und über die alternativ \\ praktizierten Partizipationsformen zu forschen und zu diskutieren.
}

Nach heftigen Auseinandersetzungen im deutschen Reichstag und auf der Straße trat am 4. Februar 1920 das sogenannte Betriebsrätegesetz in Kraft. Dieses Gesetz stellt die Geburtsstunde der betrieblichen Mitbestimmung in Deutschland dar. Zwar hatte der Betriebsrat mit den Arbeiterausschüssen einen Vorläufer. Schon die Gewerbeordnung von 1891 sah vor, in Betrieben mit mehr als 20 Mitarbeitern Arbeiterausschüsse einzurichten, allerdings auf freiwilliger Basis. Aber erst mit dem Betriebsrätegesetz wurden betriebliche Interessenvertretungen verbindlich verankert und den Betriebsräten Mitwirkungs- und Mitbestimmungsrechte in sozialen und personellen Angelegenheiten eingeräumt. Dabei war das während des Ersten Weltkriegs erlassene Gesetz über den vaterländischen Hilfsdienst (1916) ein wichtiger Wegbereiter. Dieses schrieb vor, Arbeiter- und Angestelltenausschüsse in kriegs- und versorgungswichtigen Betrieben mit mehr als 50 Beschäftigten verpflichtend einzuführen (BMAS, 2018, 15 ff.).

(C) Der/die Autor(en) 2020. Open Access: Dieser Artikel wird unter der Creative Commons Namensnennung 4.0 International Lizenz (https:// creativecommons.org/licenses/by/4.0/deed.de) veröffentlicht.

Open Access wird durch die ZBW - Leibniz-Informationszentrum Wirtschaft gefördert.

Dr. Hagen Lesch ist Leiter des Kompetenzfelds Tarifpolitik und Arbeitsbeziehungen am Institut der deutschen Wirtschaft Köln.
In $\S 1$ des 1920 erlassenen Betriebsrätegesetzes hieß es: „Zur Wahrung der gemeinsamen wirtschaftlichen Interessen der Arbeitnehmer (Arbeiter und Angestellte) dem Arbeitgeber gegenüber und zur Unterstützung des Arbeitgebers in der Erfüllung der Betriebszwecke sind in allen Betrieben, die in der Regel mindestens zwanzig Arbeitnehmer beschäftigen, Betriebsräte zu errichten." In Betrieben mit weniger als 20, aber mindestens fünf wahlberechtigten Arbeitnehmern war ein Betriebsobmann zu wählen (§ 2 Betriebsrätegesetz). Waren in einem Betriebsrat sowohl Arbeiter als auch Angestellte vertreten, waren zusätzlich jeweils ein Angestellten- und ein Arbeiterrat zu wählen (§ 6), um die jeweiligen Interessen der beiden Gruppen spezifischer vertreten zu können. Zu den Aufgaben ( $(66)$ des Betriebsrats gehörte es unter anderem

- die Betriebsleitung durch Rat zu unterstützen, um auf diese Weise für einen „möglichst hohen Stand und für möglichste Wirtschaftlichkeit der Betriebsleistungen zu sorgen",

- an der Einführung neuer Arbeitsmethoden fördernd mitzuarbeiten,

- gemeinsame Dienstvorschriften im Rahmen der geltenden Tarifverträge mit dem Arbeitgeber zu vereinbaren,

- oder auf die Bekämpfung der Unfall- und Gesundheitsgefahren zu achten. 
Dem Betriebsrat wurde ferner ein Recht auf Einsichtnahme in die Betriebsvorgänge ( $(71)$ zugestanden und ein Mitspracherecht bei einer größeren Zahl von Einstellungen oder Entlassungen eingeräumt (§ 74). Ferner hatten er oder stellvertretend die Arbeiter- und Angestelltenräte die Aufgabe, über die Einhaltung der maßgebenden Tarifverträge zu wachen. Sofern tarifliche Regelungen nicht bestehen, sollte er bei der Regelung der Löhne und sonstigen Arbeitsverhältnisse mitwirken, insbesondere bei der Festsetzung von Akkord- und Stücklohnsätzen oder der Festsetzung der Arbeitszeit (§ 78).

Insgesamt war die Aufgabenbeschreibung des Betriebsrats als kooperativ angelegt. Er sollte „nicht Interessenvertretung pur sein“ (Müller-Jentsch, 2008, 48), sondern gleichzeitig die Arbeitgeber unterstützen und sich um das Wohl des Betriebs kümmern. Nach Müller-Jentsch (2008, 49) kodifizierte das Gesetz damit „eine doppelte Loyalität des Betriebsrats gegenüber der Belegschaft auf der einen Seite und der Unternehmensleitung auf der anderen“. Den Gewerkschaften galt die betriebliche Mitbestimmung damals als wenig zukunftsweisend. Für sie war die Entsendung von Betriebsratsmitgliedern in den Aufsichtsrat, die 1922 gesetzlich eingeführt wurde, wichtiger (MüllerJentsch, 2008, 49).

Nachdem die Mitbestimmung der Arbeitnehmer 1934 durch das Gesetz zur Ordnung der nationalen Arbeit suspendiert wurde, führte der Alliierte Kontrollrat die betriebliche Mitbestimmung schon 1946 wieder ein und knüpfte dabei unmittelbar am Betriebsrätegesetz an (BMAS, 2018, 21; Niedenhoff, 2020, 13). Das gilt auch für das 1952 verabschiedete Betriebsverfassungsgesetz (BetrVG), mit dem die vom Alliierten Kontrollrat erlassene Rechtsetzung durch eine eigenständige nationale Regelung abgelöst wurde. Auch im BetrVG wird auf ein kooperatives Miteinander gesetzt: „Arbeitgeber und Betriebsrat arbeiten unter Beachtung der geltenden Tarifverträge vertrauensvoll und [...] zum Wohl der Arbeitnehmer und des Betriebs zusammen" (§ 2 Abs. 1 BetrVG). Anders als sein Vorgänger sah (und sieht) das neue Gesetz die Wahl eines Betriebsrats allerdings schon in Betrieben mit in der Regel mindestens fünf ständigen wahlberechtigten Arbeitnehmern vor. Außerdem wurde die Amtszeit der Betriebsräte von einem Jahr auf zwei Jahre verlängert. Durch die Novellierungen des BetrVG 1972 und 1988 liegt die Amtszeit inzwischen bei vier Jahren.

Eine Gegenüberstellung der Betriebsratsgröße nach dem Betriebsrätegesetz von 1920 und dem BetrVG in seiner gültigen Fassung in Tabelle 1 zeigt: Ab einer BetriebsgröBe von mehr als 100 Arbeitnehmern sieht das BetrVG für den Betriebsrat mehr Mitglieder als das Betriebsrätegesetz vor. Damals galt zudem eine Obergrenze von $30 \mathrm{Be}-$
Tabelle 1

Gesetzliche Zahl der Betriebsratsmitglieder

\begin{tabular}{rrrr}
$\begin{array}{c}\text { Arbeitnehmer } \\
1920\end{array}$ & $\begin{array}{c}\text { Betriebsratsmit- } \\
\text { glieder 1920 }\end{array}$ & $\begin{array}{c}\text { Arbeitnehmer } \\
2020\end{array}$ & $\begin{array}{c}\text { Betriebsratsmit- } \\
\text { glieder 2020 }\end{array}$ \\
\hline $5-19$ & -1 & $5-20$ & 1 \\
\hline $20-49$ & 3 & $21-50$ & 3 \\
\hline $50-99$ & 5 & $51-100$ & 5 \\
\hline $100-199$ & 6 & $101-200$ & 7 \\
\hline $200-399$ & 7 & $401-400$ & 9 \\
\hline $400-599$ & 8 & $701-1000$ & 11 \\
\hline $600-799$ & 9 & & 13 \\
\hline $800-999$ & 10 & $1001-1500$ & 15 \\
\hline $1000-1499$ & 11 & $1501-2000$ & 17 \\
\hline $1500-1999$ & 12 & Höchstzahl & -2 \\
\hline Höchstzahl & 30 & & \\
\hline
\end{tabular}

${ }^{1}$ Wahl eines Betriebsobmanns. ${ }^{2}$ Keine explizite Höchstzahl.

Quellen: § 15 Betriebsrätegesetz; § 9 BetrVG; eigene Zusammenstellung

triebsratsmitgliedern. Heute wird keine Obergrenze mehr vorgegeben. Die frühere Obergrenze von 30 Mitgliedern wurde ab einer Schwelle von 5001 Mitarbeitern überschritten. Ein weiterer Unterschied besteht darin, dass seit der Novellierung des BetrVG 1972 Betriebsräte für ihre Betriebsratsarbeit auch vollständig freigestellt werden müssen. Die Novellierung des BetrVG sah dazu vor, dass in Betrieben ab 300 Beschäftigten mindestens ein Betriebsratsmitglied freigestellt wird. Seit 2001 liegt diese Schwelle bei 200 Beschäftigten (§ 38 BetrVG).

Im Vergleich zum Betriebsrätegesetz von 1920 wurden dem Betriebsrat im BetrVG von 1952 deutlich mehr und auch differenziertere Rechte eingeräumt (Niedenhoff, 2020, 25). Im Gesetz von 1920 beschränkte sich die echte Mitwirkung vor allem auf die Vereinbarung von Dienstvorschriften mit dem Arbeitgeber, auf die Verwaltung von Pensionskassen, Werkswohnungen und sonstiger Betriebswohlfahrtseinrichtungen und auf die Überwachung der Einhaltung von Tarifverträgen (Niedenhoff, 2020, 14). Das 1952 erlassene Gesetz räumt den Arbeitnehmern hingegen zahlreiche Mitwirkungs- und Mitbestimmungsrechte in sozialen, personellen und auch wirtschaftlichen Angelegenheiten ein. Dazu gehören Beratungs- und Anhörungsrechte, Informations- und Vorschlagsrechte, Zustimmungs- und Vetorechte bis hin zu den erzwingbaren Mitbestimmungsrechten. Die erzwingbaren Mitbestimmungsrechte umfassen unter anderem die Ordnungsund Verhaltensregeln im Betrieb, kollektive Arbeitszeitregelungen, Überstunden und Kurzarbeit, Fragen der betrieblichen Lohngestaltung, der Entlohnungsgrund- 
sätze sowie der Entlohnungsmethoden, Sozialpläne bei Betriebsänderungen, die Durchführung betrieblicher Bildungsmaßnahmen oder die Regelungen zur Unfallverhütung und zum Gesundheitsdienst.

\section{Betriebliche Mitbestimmung und Partizipation heute}

Das Betriebsverfassungsgesetz gilt heute als "eine der tragenden Säulen der Arbeitsmarktordnung" in Deutschland (Klös und Stettes, 2018, 4). Auf der einen Seite kann die Institution Betriebsrat als kollektive Stimme der Arbeitnehmer dazu beitragen, die Effizienz betrieblicher Abläufe zu steigern. Auf der anderen Seite kann der Betriebsrat danach streben, die Unternehmensgewinne zugunsten der Arbeitnehmer umzuverteilen. Das kann zu Konflikten führen (Stettes, 2010; Jirjahn und Smith, 2017). Empirische Untersuchungen für Deutschland kommen zu dem Ergebnis, dass die betriebliche Mitbestimmung für Unternehmen wie Arbeitnehmer zu einem Mehrwert (Winwin-Situation) führen kann. Das hängt allerdings entscheidend davon ab, ob das Management der Institution "Betriebsrat" aufgeschlossen gegenübersteht und beide Seiten miteinander kooperieren (Jirjahn und Smith, 2017). Wichtig ist dabei, Probleme frühzeitig zu lokalisieren und einen Konsens mit dauerhaften Lösungen herzustellen (Niedenhoff, 2020, 53).

Eine aktuelle Umfrage unter Personalverantwortlichen aus dem Jahr 2018 zeigt, dass dies trotz aller Interessenunterschiede gelingt: In $95 \%$ aller betrieblichen Entscheidungen schaffen es Unternehmensleitung und Betriebsrat, einen Konsens herzustellen und nur in $5 \%$ müssen Entscheidungen in der Regel gegen den Betriebsrat durchgesetzt werden (Schneider et al., 2019, 118). Wann es zu einer eher kooperativen Zusammenarbeit kommt und wann Konflikte überwiegen, ist empirisch noch nicht abschließend untersucht worden. Es liegt aber die Vermutung nahe, "dass individuelle Faktoren oder Rahmenbedingungen wie Dauer und Beständigkeit der Zusammenarbeit auf die Kooperationsfähigkeit wirken könnten. Betriebsräte und Management interagieren durch Personen, bei denen Lernprozesse eine Rolle spielen" (Schneider et al., 2019, 122).

Trotz dieser guten Zusammenarbeit und der dadurch potenziell erreichbaren Vorteile ist die Reichweite der betrieblichen Mitbestimmung in Deutschland beschränkt. Wie schon im ersten Betriebsrätegesetz von 1920 gilt auch heute: Die Arbeitnehmer haben ein Recht dazu, Betriebsräte zu wählen, sie sind dazu aber nicht verpflichtet. Ein Betriebsrat existiert gerade mal in $9 \%$ aller Betriebe mit mindestens fünf Mitarbeitern (Ellguth und Kohaut, 2019, 295). Vor allem in kleineren und mittleren Betrieben wird selten ein Betriebsrat gewählt. In Betrieben mit
Tabelle 2

Betriebe mit Betriebsrat in Deutschland in $\%$

Westdeutschland Ostdeutschland

$1996 \quad 2018 \quad 1996 \quad 2018$

Betriebe mit

\begin{tabular}{lrrrr}
\hline 5 bis 50 Beschäftigten & 8 & 5 & 7 & 6 \\
\hline 51 bis 100 Beschäftigten & 49 & 32 & 46 & 36 \\
\hline 101 bis 199 Beschäftigten & 71 & 53 & 63 & 45 \\
\hline 200 bis 500 Beschäftigten & 84 & 73 & 85 & 69 \\
\hline 501 und mehr Beschäftigten & 91 & 87 & 81 & 88 \\
\hline Alle & 12 & 9 & 11 & 10 \\
\hline
\end{tabular}

Quellen: Ellguth und Trinczek (2014), Ellguth und Kohaut (2019); eigene Zusammenstellung.

mehr als 500 Mitarbeitern ist der Betriebsrat hingegen Standard: Sowohl in Westdeutschland als auch in Ostdeutschland verfügen knapp neun von zehn Betrieben dieser Größe über einen Betriebsrat (vgl. Tabelle 2). Aufgrund dieses Betriebsgrößeneffekts ist die Reichweite der betrieblichen Mitbestimmung unter den Beschäftigten höher. So arbeiten in Westdeutschland $42 \%$ und in Ostdeutschland $35 \%$ der Beschäftigten in Betrieben mit einem Betriebsrat. Vorbehalte gegenüber der Einrichtung eines betrieblichen Mitbestimmungsgremiums bestehen vor allem in inhabergeführten Unternehmen (Behrens und Dribbusch, 2014, 147; Jirjahn und Smith, 2017, 36). Dies dürfte damit zusammenhängen, dass Mitwirkung und Mitbestimmung von Arbeitnehmern in Unternehmen mit einer patriarchalischen Unternehmensführung, die durch einen „Herr im Hause“-Standpunkt geprägt ist, schlecht zusammenpassen. Die Firmenchefs sind offenbar der Ansicht, sie könnten für das Wohl des Betriebs und seiner Arbeitnehmer allein besser sorgen. Hinzu kommt (vgl. Tabelle 2), dass die Verbreitung von Betriebsräten in mittelgroßen Betrieben (mit 51 bis 500 Beschäftigten) seit Mitte der 1990er Jahre überdurchschnittlich zurückgegangen ist (Ellguth und Trinczek, 214, 172).

Damit bestehen in Deutschland unterschiedliche Welten der Partizipation von Beschäftigten. Es gibt eine Welt der Großunternehmen, in der Betriebsräte ein fester Bestandteil der Arbeitsbeziehungen und der Unternehmenskultur sind. Die recht hohe Wahlbeteiligung - sie lag bei der letzten Betriebsratswahl 2018 in Betrieben, die einen Betriebsrat gewählt haben, bei $75 \%$ (Kestermann et al., 2018) - ist ein Indiz dafür, dass die Arbeitnehmer dem Betriebsrat wegen seiner Mitwirkungs- und Mitbestimmungsrechte einen hohen Stellenwert beimessen (Niedenhoff, 2020, 37). Auf der anderen Seite gibt es allerdings eine wesentlich größere Welt des inhaberge- 
führten Mittelstands, dem betriebliche Mitbestimmung eher fremd ist. Hinzu kommt, dass dies unweigerlich die Frage aufwirft, warum die Reichweite der betrieblichen Mitbestimmung so gering ist. Offenbar sehen viele Arbeitnehmer keine Notwendigkeit, Betriebsräte zu wählen. In Einzelfällen kann es zwar vorkommen, dass Betriebsratswahlen arbeitgeberseitig behindert wurden. Eine tiefere Untersuchung dieses Phänomens zeigt aber, dass dies „kein stilbildendes Merkmal der deutschen Arbeitsbeziehungen" ist (Behrens und Dribbusch, 2014, 147). Es erklärt jedenfalls kaum, warum Arbeitnehmer in kleinen und mittleren Unternehmen keine Betriebsräte gründen. Empirische Untersuchungen kommen zu dem Ergebnis, dass der Rückgang in den mittelgroßen Unternehmen weniger auf veränderte Einstellungen der Arbeitnehmer zurückzuführen sei. Verantwortlich dafür sind eher strukturelle Veränderungen wie die Tatsache, dass sich der allgemeine Rückgang der Tarifbindung, der Trend zu kleineren Betriebseinheiten sowie die Tertiarisierung negativ auf die Reichweite der betrieblichen Mitbestimmung auswirken (Ellguth und Trinczek, 2014).

Allerdings sollte man die Partizipation von Arbeitnehmern nicht auf die Interessenvertretung durch einen Betriebsrat verkürzen. Dies würde dem Umstand nicht gerecht, dass sich vielerorts alternative Formen der Mitarbeitervertretung abseits der Betriebsverfassung finden (Schnabel und Wagner, 2001; Stettes, 2010). Immerhin existieren in $18 \%$ aller Betriebe alternative betriebliche Vertretungsorgane wie Mitarbeitervertretungen, Belegschaftssprecher oder „Runde Tische" (Ellguth und Kohaut, 2019). Solche Institutionen mögen weniger stabil als Betriebsräte sein. Sie etablieren sich aber unter spezifischen Bedingungen, also dann, wenn es aus Sicht der Beschäftigten und/oder der Unternehmensleitung sinnvoll erscheint. Auch sie räumen den Beschäftigten eine Partizipation an betrieblichen Entscheidungsprozessen ein. Das kann dazu führen, dass sich die Motivation für die Wahl von Betriebsräten und alternativen Vertretungsorganen unterscheidet. Die empirische Evidenz legt nahe, dass für Betriebsräte besonders die Rolle als Schutzeinrichtung wichtig ist, während gemeinsam vom Arbeitgeber und von Mitarbeitervertretern besetzte Beratungs- und Entscheidungsgremien die Aktivierung des Wissenspotenzials und der Informationsvorsprünge der Beschäftigten erleichtern (Stettes, 2010, 208). Alternative Vertretungsorgane sind daher attraktiv, um Arbeitnehmer an betrieblichen Entscheidungsprozessen partizipieren zu lassen (Schnabel und Wagner, 2001, 460).

Alternative Formen der Beteiligung können nicht nur betriebswirtschaftlich effizient sein. Eine Auswertung von Daten des Sozio-oekonomischen Panels (SOEP) aus dem Jahr 2016 - in diesem Jahr wurde letztmals danach gefragt, ob eine Person in einem Betrieb mit Betriebs- oder
Tabelle 3

Arbeitszufriedenheit der Arbeitnehmer, 2016

in \%

\begin{tabular}{lccc}
$\begin{array}{l}\text { Zufrieden- } \\
\text { heitsskala }\end{array}$ & $\begin{array}{c}\text { Arbeitnehmer in } \\
\text { Firmen ohne Betriebs- } \\
\text { oder Personalrat }\end{array}$ & $\begin{array}{c}\text { Arbeitnehmer in } \\
\text { Firmen mit Betriebs- } \\
\text { oder Personalrat }\end{array}$ & $\begin{array}{c}\text { Alle } \\
\text { Arbeitneh- } \\
\text { mer }\end{array}$ \\
\hline 0 bis 3 & 6,2 & 4,8 & 5,4 \\
\hline 4 bis 6 & 21,6 & 21,9 & 21,8 \\
\hline 7 bis 10 & 72,2 & 73,1 & 72,8
\end{tabular}

${ }^{1}$ In Abhängigkeit von der Existenz eines Betriebs- oder Personalrats. Berücksichtigt wurden alle Arbeitnehmer und Auszubildenden, die jünger als 66 Jahre alt sind. ${ }^{2}$ Die Skala der Zufriedenheit mit der Arbeit reicht von 0 (= ganz und gar unzufrieden) bis 10 (= ganz und gar zufrieden).

Quelle: SOEP (Welle 33, 2016); eigene Berechnungen.

Personalrat (in öffentlichen Unternehmen) arbeitet - zeigt auch: Die Arbeitszufriedenheit liegt in der Gruppe der Arbeitnehmer, die in einem Betrieb mit einem Betriebs- oder Personalrat arbeiten, nicht signifikant höher als in der Gruppe der Arbeitnehmer, die in einem Betrieb ohne Betriebs- oder Personalrat arbeiten. Unterteilt man die auf einer Skala von 0 (ganz und gar unzufrieden) bis 10 (ganz und gar zufrieden) gemessene Zufriedenheit in drei Gruppen ( 0 bis 3 für die Unzufriedenen, 4 bis 6 für die weder Unzufriedenen noch Zufriedenen und 7 bis 10 für die Zufriedenen) (vgl. Tabelle 3), ergeben sich - für die jeweiligen Vertretungsformen - ähnliche Anteile. Im Durchschnitt liegt die Arbeitszufriedenheit von Arbeitnehmern in Betrieben mit Betriebsrat bei 7,21. Arbeitnehmer in Betrieben ohne Betriebsrat kommen auf einen Wert von 7,25. Dieser ohnehin nur marginale Unterschied ist statistisch nicht signifikant.

\section{Perspektiven der betrieblichen Mitbestimmung}

Dennoch wird aktuell intensiv darüber diskutiert, ob das BetrVG angesichts der Digitalisierung der Arbeitswelt modernisiert und seine Reichweite vergrößert werden muss. Im Koalitionsvertrag zwischen CDU, CSU und SPD betonen die Regierungsparteien, dass der betrieblichen Mitbestimmung im digitalen Wandel große Bedeutung zukomme (Koalitionsvertrag 2018). Daher soll das allgemeine Initiativrecht der Betriebsräte für Weiterbildung gestärkt werden. Außerdem wollen die Regierungsparteien die Gründung und die Wahl von Betriebsräten erleichtern, indem das vereinfachte Wahlverfahren für alle Betriebe mit 5 bis 100 wahlberechtigten Arbeitnehmerinnen und Arbeitnehmern verpflichtend wird. Für Betriebe mit 101 bis 200 Wahlberechtigten soll die Wahl zwischen dem vereinfachten und dem allgemeinen Wahlverfahren ermöglicht werden. 
Der Deutsche Gewerkschaftsbund (DGB) hat schon 2016 eine Offensive „Mitbestimmung" gestartet. Im Rahmen derer fordert er, dass die Betriebsverfassung gestärkt und weiterentwickelt wird (DGB, 2016). Um die Reichweite der Betriebsverfassung zu erhöhen und die Gründung eines Betriebsrats zu erleichtern, soll das 2001 für Kleinbetriebe mit bis zu 50 Mitarbeitern eingeführte vereinfachte Wahlverfahren künftig für Betriebe mit bis zu 100 Mitarbeitern ausgedehnt werden. Dabei soll auch der Kündigungsschutz für (potenzielle) Betriebsräte verbessert werden. Darüber hinaus soll die Mitbestimmung des Betriebsrats bei Datenschutz, Weiterbildung und Ausgliederung erweitert werden. Dabei soll er auch leichter externen Sachverstand hinzuziehen können. Schließlich sollen der Arbeitnehmer- und der Betriebsbegriff auf neue Formen der globalen, digitalen Arbeit wie Clickworking erweitert werden.

Ob eine Ausweitung des vereinfachten Wahlverfahrens zu einer höheren Reichweite der betrieblichen Mitbestimmung führen wird, ist mehr als fraglich. Nach dem vereinfachten Wahlverfahren kann ein Betriebsrat in einer kürzeren Frist gewählt werden (DGB, 2009). Es ist aber juristisch umstritten, ob die Wahl eines Betriebsrats dadurch tatsächlich erleichtert wird. Das 2001 eingeführte Verfahren hat in den betroffenen Betrieben jedenfalls keinen Gründungsboom ausgelöst, wie die geringe Betriebsrätedichte unter den Betrieben mit bis zu $50 \mathrm{Be}-$ schäftigten zeigt (vgl. Tabelle 2). Auch künftig dürfte für das Gründungsgeschehen eher entscheidend sein, welche Form der Partizipation die Beschäftigten in den jeweiligen Betrieben bevorzugen.

\section{Digitalisierung der Arbeitswelt: Auswirkungen}

Hinzu kommt: Empirische Untersuchungen zeigen, dass die Digitalisierung bislang keinen systematischen Einfluss auf die Verbreitung von Betriebsräten hatte (Schneider et al., 2019, 112). Daher scheint auch die Befürchtung, wonach die Digitalisierung zu einer Erosion der verfassten betrieblichen Mitbestimmung führen wird, "derzeit eher unbegründet“" (Schneider et al., 2019, 113). Umgekehrt hängt aber auch die Einführung digitaler Technologien nicht davon ab, ob ein Betriebsrat vorhanden ist oder nicht. Die Digitalisierung liefert nach dem derzeitigen empirischen Kenntnisstand keine Begründung dafür, die Einrichtung von Betriebsräten mit zusätzlichen Impulsen zu fördern. Die Digitalisierung hat auch keinen negativen Einfluss auf die Kooperationsbereitschaft zwischen Unternehmensleitung und Betriebsräten, die eine Anpassung des BetrVG notwendig erscheinen lassen.

Bevor die Mitwirkungs- und Mitbestimmungsrechte des Betriebsrats aufgrund der Herausforderungen durch die
Digitalisierung ausgeweitet werden, ist ohnehin sorgfältig zu prüfen, welche Rechte das BetrVG bereits einräumt. Wie die Digitalisierung die Arbeitswelt verändern wird, ist heute nur schwer abschätzbar (Hammermann und Stettes, 2019). Unbestritten ist, dass die Bildungsanforderungen an die Beschäftigten erheblich steigen werden und eine Flexibilisierung von Arbeitszeit und -ort stattfinden wird (Niedenhoff, 2020, 57). Um die Beschäftigungsfähigkeit der Arbeitnehmer in Hinblick auf den wichtigen Ansatzpunkt „Bildung“ zu erhalten, sind bereits wichtige Mitbestimmungsrechte im BetrVG implementiert (Niedenhoff, 2020, 59). So schreibt § 96 Abs. 1 BetrVG vor, dass der Arbeitgeber auf Verlangen des Betriebsrats den Berufsbildungsbedarf zu ermitteln hat. Fragen der Berufsbildung der Arbeitnehmer des Betriebs muss der Arbeitgeber mit dem Betriebsrat zusammen beraten. Gemäß § 97 Abs. 2 BetrVG bestimmt der Betriebsrat bei der Einführung von Maßnahmen der betrieblichen Berufsbildung mit. Kommt eine Einigung nicht zustande, entscheidet die Einigungsstelle. In § 98 wird dem Betriebsrat die Mitbestimmung bei der Durchführung betrieblicher Bildungsmaßnahmen eingeräumt. Damit der Betriebsrat seine Aufgaben erfüllen kann, verpflichtet § 92 BetrVG den Arbeitgeber, den Betriebsrat über die Personalplanung und den Personalbedarf sowie über die sich daraus ergebenden personellen Maßnahmen und Maßnahmen der Berufsbildung anhand von Unterlagen rechtzeitig zu unterrichten. Der Betriebsrat erhält also bereits nach geltendem Recht hinreichend Einsicht in die Personalplanung, um frühzeitig die zu erwartenden Soll-Qualifikationsanforderungen mit dem Ist-Zustand der Qualifikation abgleichen und entsprechende Maßnahmen einfordern zu können.

Der Betriebsrat kann nach geltendem Recht auch schon umfassend an der Flexibilisierung von Arbeitszeit und -ort mitwirken (Niedenhoff, 2020, 50 f.). Hinzu kommen seine Unterrichtungs- und Beratungsrechte über die wirtschaftliche und finanzielle Lage des Unternehmens, die Produktions- und Absatzlage, das Produktions- und Investitionsprogramm, Rationalisierungsvorhaben, die Einführung neuer Arbeitsmethoden, die Einschränkung oder Stilllegung von Betrieben oder über die Änderung der Betriebsorganisation oder des Betriebszwecks.

Das schließt nicht aus, das BetrVG bei bestimmten Regelungen der veränderten Arbeitswelt anzupassen. So könnten neue Beschäftigungsarten wie Homeoffice, Telearbeit, Crowdworking oder Solo-Selbständige eine Bearbeitung des $\S 5$ BetrVG notwendig machen (Niedenhoff, 2020, 61). In diesem Paragrafen wird definiert, was ein Arbeitnehmer im Sinne des Gesetzes ist. Hier wäre zu klären, wer ein Arbeitnehmer ist, wer eine arbeitnehmerähnliche Position innehat und wie ein Betriebsrat SoloSelbständige oder Crowdworker künftig vertreten kann. 
Bisher liegen allerdings weder belastbare empirische Erkenntnisse zur Verbreitung von Crowdworking oder Plattformarbeit noch zum Prekaritätspotenzial der betroffenen Personen vor (Schäfer, 2019). Weitere Ansatzpunkte einer Modernisierung des BetrVG könnte die Möglichkeiten des Betriebsrats betreffen, künftig moderne Kommunikationsmethoden wie Online-Wahlen, Betriebsratssitzungen per Videokonferenz oder virtuelle Betriebsversammlungen anwenden zu können (Thüsing, 2020).

\section{Fazit}

Zusammenfassend lässt sich festhalten, dass sich 100 Jahre nach dem ersten Betriebsrätegesetz in Deutschland unterschiedliche Partizipationsformen entwickelt haben. Während in den Großunternehmen die betriebliche Mitbestimmung fest verankert ist, wird im Mittelstand eher auf weniger institutionalisierte Beteiligungsformen gesetzt. Sofern - wovon zumindest in der überwiegenden Mehrheit der Betriebe auszugehen ist - die Arbeitnehmer frei darüber entscheiden können, ob sie einen Betriebsrat wählen wollen oder nicht, stellt dies eine Mischung dar, aus der sich keine Neujustierung der gesetzlichen Rahmenbedingungen ableiten lässt. Zudem ist derzeit auch noch nicht erkennbar, dass die Digitalisierung die Betriebsräte vor Herausforderungen stellt, auf die sie mithilfe der bereits in der Betriebsverfassung angelegten Befugnisse nicht angemessen reagieren können. Das schließt Anpassungen in Einzelfragen nicht aus. Auf jeden Fall sollte das Jubiläum Anlass dafür sein, intensiver über die Zukunft der deutschen Betriebsverfassung und über die alternativ praktizierten Partizipationsformen zu forschen und zu diskutieren.

\section{Literatur}

Behrens, M. und H. Dribbusch (2014), Arbeitgebermaßnahmen gegen Betriebsräte: Angriffe auf die betriebliche Mitbestimmung, WSI-Mitteilungen, 67(2), 140-148.

Bundesministerium für Arbeit und Soziales (BMAS) (2018), Mitbestimmung - eine gute Sache. Alles über die Mitbestimmung und ihre rechtlichen Grundlagen, https://www.bmas.de/SharedDocs/Downloads/DE/PDF-Publikationen/a741-mitbestimmung-ein-gutes-unternehmen.pdf?__blob=publicationFile (20. Januar 2020).
Deutscher Gewerkschaftsbund (DGB) (2009), Wahlleitfaden Vereinfachtes Wahlverfahren - Arbeitshilfe für Wahlvorstände, Berlin.

Deutscher Gewerkschaftsbund (DGB) (2016), Offensive Mitbestimmung - Vorschläge zur Weiterentwicklung der Mitbestimmung, Beschluss des DGB-Bundesvorstands vom 12.6.2016, Berlin.

Ellguth, P. und S. Kohaut (2019), Tarifbindung und betriebliche Interessenvertretung: Ergebnisse aus dem IAB-Betriebspanel 2018, WSIMitteilungen, 72(4), 290-297.

Ellguth, P. und R. Trinczek (2014), Erosion der betrieblichen Mitbestimmung - Welche Rolle spielt der Strukturwandel?, WSI-Mitteilungen, 67(3), 172-182.

Jirjahn, U. und S. C. Smith (2017), Nonunion Employee Representation: Theory and the German Experience with Mandated Works Councils, IZA-Discussion Papers, 11066, Bonn.

Hammermann, A. und O. Stettes (2019), Arbeitswelt im digitalen Wandel - Empirische Evidenz und Gestaltungsaufgaben, HMD Praxis der Wirtschaftsinformatik, 56(4), 706-720.

Kestermann, C., H. Lesch und O. Stettes (2018), Betriebsratswahlen 2018: Ergebnisse der IW-Betriebsratswahlbefragung, IW-Trends, 45(4), 79-93.

Klös, H.-P. und O. Stettes (2018), Befunde zu Betriebsräten und Betriebsratswahlen. Stellungnahme für den Ausschuss für Arbeit und Soziales des Deutschen Bundestages, IW-Report, 26, https://www.iwkoeln.de/fileadmin/user_upload/Studien/Report/PDF/2018/IW-Report_2018_26_ Stellungnahme_Betriebsratswahlen.pdf (20. Januar 2020).

Koalitionsvertrag (2018), Ein neuer Aufbruch für Europa. Eine neue Dynamik für Deutschland. Ein neuer Zusammenhalt für unser Land. Koalitionsvertrag zwischen CDU, CSU und SPD für die 19. Wahlperiode, https://www.cdu.de/system/tdf/media/dokumente/koalitionsvertrag_2018.pdf?file=1 (21. Januar 2020).

Müller-Jentsch, W. (2008), 1918 - 1952. Mitbestimmung für eine neue Wirtschaftsordnung nutzen, Die Mitbestimmung, 54(1+2), 47-51.

Niedenhoff, H.-U. (2020), 100 Jahre betriebliche Mitbestimmung in Deutschland, Münster.

Schäfer, H. (2019), Crowdwork und Plattformarbeit in Deutschland, IWKurzbericht, 79, https://www.iwkoeln.de/fileadmin/user_upload/Studien/Kurzberichte/PDF/2019/IW-Kurzbericht_2019_Crowdwork.pdf (22. Januar 2020).

Schnabel, C. und J. Wagner (2001), Verbreitung und Bestimmungsgründe verschiedener Formen der Arbeitnehmerpartizipation in Industriebetrieben, Industrielle Beziehungen, 8(4), 445-462.

Schneider, H., O. Stettes und S. Vogel (2019), Betriebliche Arbeitsbeziehungen und Transformationsprozesse, IW-Trends, 46(3), 109-125.

Sozio-oekonomische Panel (SOEP) (2016), Erhebungsinstrumente 2016 (Welle 33): Personenfragebogen Kurzfassung (Lücke), Altstichproben; https://www.diw.de/de/diw_01.c.549772.de/soepsurveypapers/ soep_2016_erhebungsinstrumente_2016_welle_33_des_sozio_ oekonomischen_panels_personenfragebogen_kurzfassung_luecke_ altstichproben.html (23. April 2020).

Stettes, O. (2010), Betriebsräte und alternative Mitbestimmungsformen in der Industrie und deren Verbundbranchen, Sozialer Fortschritt, 59(8), 199-209.

Thüsing, G. (2020), 100 Jahre Betriebsrätegesetz, Mut zur Reform, Frankfurter Allgemeine Zeitung vom 25.1.2020, https://www.faz.net/aktuell/ wirtschaft/unternehmen/warum-das-betriebsraetegesetz-reformiertwerden-sollte-16597278.html (23. April 2020).

Title: 100 Years of the Works Council Act and Current Participation of Employees in Germany

Abstract: TIn Germany, the first Works Council Act came into force at the beginning of 1920. After it was suspended in 1934, the Allied Control Council reintroduced co-determination in 1946 and it was finally established after several reforms. However, while co-determination is firmly anchored in large companies, medium-sized companies tend to opt for less institutionalised forms of participation. However, the change in work organisation through crowdworking, teleworking and solo self-employment poses new challenges for company cooperation. At present, however, it is not yet apparent that works councils are unable to respond appropriately to these challenges with the powers already laid down in the works constitution. The anniversary should be an occasion for more intensive research and discussion on the future of the German works constitution and alternative forms of participation that are being practiced.

JEL Classification: J50, J53, J83 